\title{
Liebeskarotte / Wortgarrotte - Beobachtungen aus dem Folterhaus der Sprache
}

\begin{abstract}
Der Essay spürt dem Zusammenhang von Sprache und Liebe nach und bedient sich hierbei der psychoanalytischen Theorie, die den libidinösen Aspekt der Sprache stets hervorgehoben hat: Sprache ist das mächtigste Medium des Eros und zugleich auch fundamental mit dem Todestrieb verbunden - das „Unbehagen in der Kultur“ lässt sich immer auch als ein Unbehagen an der Liebe lesen. Ein wichtiges Interface zwischen Sprache und Liebe bildet der kulinarische Komplex: In Samuel Becketts Roman Der Namenlose wird die Frage nach dem Innen und Außen, nach dem logo-libidinösen Verschlingungszusammenhang mit dem Leitmotiv der Nahrungsaufnahme bzw. deren Verweigerung verquickt. Es ist der Diskurs eines Hungerkünstlers, der paradigmatisch für das dunkle Begehren der Sprache steht - und für die Kritik an der Kultur als aufgehobener Kannibalismus. Was die avantgardistische Literatur und die psychoanalytische Theorie in den Fokus rücken, ist das „Folterhaus der Sprache“, in dem der Mensch, von der Sprache erfasst und gepeinigt, sein Begehren unaufhörlich artikulieren muss. Die Wahrheit (der Liebe) zeigt sich aber erst dort, wo die Sprache sich ihrer (ideologischen) Entstellungsarbeit bewusst wird und - wie es Roland Barthes nannte - zu einer „Agape der Syntax“ findet. Literatur entpuppt sich im kulinarischen Kontext von Liebe und Logos als niemals abzuschließende Kronos-Dekonstruktion.
\end{abstract}

Keywords: Liebe; Sprache; Nahrung; Hunger; Psychoanalyse; Beckett; Freud; Lacan; Kronos; Namenlose

Die Sprache der Liebe - die Liebe zur Sprache - Sprache und Liebe: Wenn wir nach der Verbindung von Sprache und Liebe fragen, nach der erotischen Macht der Worte, muss zunächst in Frage gestellt werden, was das sei, die Liebe. Was heißt Liebe? Wie lässt sich das Wort Liebe definieren? Wir wollen es hier mit einer psychoanalytischen Annäherung versuchen, gerade deshalb, weil im psychoanalytischen Diskurs nach Freud die allerweiteste Verwendung des Liebes-Begriffs im Mittelpunkt steht. Freud betonte in seinem Werk immer wieder - weil ihm wiederholt der Vorwurf der Pansexualität gemacht wurde -, dass in seiner Trieblehre „Libido“ genau in dem offenen Bedeutungsspektrum zu begreifen sei, in dem auch im deutschen Sprachgebrauch das Wort „Liebe“ schillert: vom rohsten Sexualakt, dem Akt des „Liebe-Machens“, über die zärtliche Zuneigung zu den

2 Open Access. (c) 2021 Rainer Just, published by De Gruyter. (c) BY-NC-ND This work is licensed under the Creative Commons Attribution-NonCommercial-NoDerivatives 4.0 International License.

https://doi.org/10.1515/9783110642032-007 
Eltern und Geschwistern bis zu den sublimsten, vergeistigtsten Formen, etwa die christliche Agape, reicht der Spielraum der Denotationen (vgl. Freud 1999g, 98 f.). Nur in dieser Hinsicht lässt sich auch Jacques Lacans Bemerkung verstehen, dass in der Psychoanalyse immer nur über die Liebe gesprochen werde, ja dass das Sprechen über die Liebe im Grunde ihr einziger Gegenstand sei: „Von Liebe zu sprechen, in der Tat, nichts anderes tut man im analytischen Diskurs.“ (Lacan 1986, 90) Die Psychoanalyse stellt die Frage nach dem Begehren - désir, desire -, nach dem Wunsch - wie es noch bei Freud geheißen hat -, dem Wunsch nach Liebe, den jeder Mensch unaufhörlich und in vielfältig verwickelten Formen artikuliert, von Beginn an und bis zuletzt, bis zum Tod, und sogar darüber hinaus. Um die Artikulation des Begehrens vom Bedürfnis zu unterscheiden, gibt die Psychoanalyse gerne ein anschauliches Beispiel aus der frühkindlichen Genese: Das Neugeborene schreit, weil es Hunger hat, und bekommt die Mutterbrust gereicht, die den Hunger stillt und das Bedürfnis nach Nahrung befriedigt, doch die Brust gibt mehr als Milch, sie spendet auch Wärme, sie vermittelt das Gefühl von Geborgenheit und Zuspruch, begleitet von der Erfahrung einer sanften, beruhigenden Stimme, die, während das Kind an der Brust saugt, zu ihm spricht. Durch die zärtliche Fürsorge, die es so erfährt, verwandelt sich der Schrei nach Nahrung in einen Schrei nach Liebe, in einen Anspruch auf mehr - mehr als bloß die nackte Bedürfnisbefriedigung, die Stillung des Hungers. Das Menschenkind hat zu begehren begonnen: Es begehrt, vom anderen beachtet, umsorgt, verwöhnt, geliebt zu werden. Begehren ist somit, im starken psychoanalytischen Sinn, immer Anspruch auf Liebe, ist Begehren nach dem Begehren des anderen. Und mit dem Begehren, das nicht nur verlangt, was es unmittelbar braucht, sondern auch nach dem sich sehnt, was darüber hinaus Lust bereitet, kommt die Sprache ins Spiel. Die Liebe spricht. Die Liebe sagt immer: Sprich mit mir! Die Liebe, das emphatische Begehren, verlangt nach der Sprache des anderen. Es verlangt sie im doppelten Sinn: Das Begehren wird durch die Sprache hervorgerufen und benötigt die Sprache, um sich am Leben, am Sehnen, am Laufen zu halten. Im Zusammenhang mit der Frage nach der Angst - und Angst ist für Freud grundsätzlich „Angst vor dem Liebesverlust“ (Freud 1999h, 483) - erzählt Freud folgende erhellende Begebenheit: „Die Aufklärung über die Herkunft der kindlichen Angst verdanke ich einem dreijährigen Knaben, den ich einmal aus einem dunklen Zimmer bitten hörte: ,Tante, sprich mit mir; ich fürchte mich, weil es so dunkel ist.' Die Tante rief ihn an: ,Was hast du denn davon? Du siehst mich ja nicht. 'Das macht nichts', antwortete das Kind, ,wenn jemand spricht, wird es hell.““ Und Freud folgert: „Er fürchtete sich also nicht vor der Dunkelheit, sondern weil er eine geliebte Person vermißte, und konnte versprechen sich zu beruhigen, sobald er einen Beweis von deren Anwesenheit empfangen hatte.“ (Freud 1999c, 126) Die Sprache, hier blitzt es deutlich auf, ist nicht nur das zentrale Instrument der Auf- 
klärung, der verbindlichen Erkenntnis von Wahrheit, sie ist auch - oder besser gesagt: gerade deswegen - das mächtigste Medium der Liebe. Freud setzte bedingungslos auf die grenzenlose Macht der Sprache, sowohl im Guten als auch im Bösen:

\footnotetext{
Worte waren ursprünglich Zauber, und das Wort hat noch heute viel von seiner alten Zauberkraft bewahrt. Durch Worte kann ein Mensch den anderen selig machen oder zur Verzweiflung treiben, durch Worte überträgt der Lehrer sein Wissen auf die Schüler, durch Worte reißt der Redner die Versammlung der Zuhörer mit sich fort und bestimmt ihre Urteile und Entscheidungen. Worte rufen Affekte hervor und sind das allgemeine Mittel zur Beeinflussung der Menschen untereinander. (Freud 1999f, 10)
}

Die magische Allmacht der Sprache, ihr Witz und Aber-Witz, „Verbindungsbrücken von überallher überallhin“ zu schlagen, wie es in der Schrift über Deckerinnerungen heißt (Freud 1999a, 549), ist eine zutiefst erotische. Denn wenn wir zur Ausgangsfrage zurückkehren, zur Frage nach der Definition von Liebe, dann ist die grundlegendste Ebene die des Eros, jenes Grundprinzips des Lebens, das danach strebt, Verbindungen einzugehen, um durch den Zusammenschluss mit anderen Objekten höhere, komplexere Einheiten zu schaffen. Aus zwei mach eins! - Das ist die Zauberformel des Eros, das Simsalabim der Liebe, der Hokuspokus der Kopulation. Bekannterweise wird aber dieses „Liebesstreben“ (Freud 1999h, 478) von einem Destruktionstrieb kontrastiert, der auf die Regression, auf die gewaltsame Bewahrung der Eigenexistenz aus ist und die höheren Einheiten zu zerstören sucht. Hier kommt Freuds berühmter Todestrieb ins Spiel, der die libidinösen Triebe unterwandert bzw. in ein „Jenseits des Lustprinzips“ führt. Die „Kulturentwicklung“, schreibt Freud in „Das Unbehagen in der Kultur“, sei gezeichnet vom „Kampf zwischen Eros und Tod, Lebenstrieb und Destruktionstrieb“. Grundsätzlich, fährt er fort, sei das „Programm der Kultur“ ein „Werk des Eros“, „der vereinzelte menschliche Individuen, später Familien, dann Stämme, Völker, Nationen zu einer großen Einheit, der Menschheit, zusammenfassen wolle.“ Die „Menschenmengen sollen libidinös aneinander gebunden werden“, was aber immer nur zum Teil gelinge, da es auch den „natürliche[n] Aggressionstrieb der Menschen“ gebe, „die Feindseligkeit eines gegen alle und aller gegen einen“ (Freud 1999h, 481). Das Projekt der Kultur und der Aufklärung arbeitet deshalb, wie Freud es nennt, immer mit „Liebesprämien“ (Freud 1999d, 335): „Durch die Zumischung der erotischen Komponenten werden die eigensüchtigen Triebe in soziale verwandelt.“ (Freud 1999d, 333) So wie jede „soziale Angst“, laut Freud, „Angst vor dem Liebesverlust“ ist, so ist auch jede soziale Hoffnung ein Versprechen von Liebesgewinn. In diesem Sinn ist auch jedes „Unbehagen in der Kultur“ ein Unbehagen an der Liebe. Und man kann hinzufügen: auch ein Unbehagen an der Sprache, diesem mächtigsten Medium des Eros. 
Der Liebe entkommt man nicht, genauso wenig wie der Sprache. „Die Liebe ist eine Karotte, die immer zieht“, heißt es in Samuel Becketts Roman „Der Namenlose“: „L'amour, voilà une carotte qui n’a jamais raté“. In der Übersetzung von Elmar Tophoven, die Beckett autorisiert hat: „Die Liebe ist [...] eine Möhre, die immer zieht, ich habe immer wieder irgendeine vernaschen müssen." (Beckett 1995, 41) Wer das sagt, ist der Namenlose, der nicht aufhören kann zu sprechen, der nicht aufhören kann zu begehren, weil der, der spricht, immer auch der ist, der begehrt, der weiter danach verlangt, gehört, erhört, geliebt zu werden, egal wie zerstört die Sprache schon scheint oder der Körper, dieser Torso mit Kopf, aus dem die eigene Stimme kommt und in dem die fremden Stimmen widerhallen, die Kakophonie der verinnerlichten anderen. „Der Diskurs muß weitergehen“ (Beckett 1995, 10 f.), bekennt der Namenlose, er muss immer weitergehen, weil das Ziel, zu schweigen - Hamlets Rest - lockt und weil dieses Ziel im Leben, solange es, von der Liebeskarotte angezogen, „immer vorwärts dringt“, unerreichbar bleibt. Die „Wut zu sprechen“ (Beckett 1995, 83), die den Namenlosen antreibt, respondiert todesgetrieben auf die unaufhörliche Libido, als die Freud die fortwuchernden „Kraftäußerungen des Eros“ (Freud 1999h, 480) bezeichnet hat: „schon wieder Leben, immerzu und überall Leben“ (Beckett 1995, 86). Es lebt. Es denkt. Es spricht. Es isst. Der Mensch ist ein unheimliches „Denkfleisch“ (Beckett 1995, 92), ist die Hegel'sche „Amphibie“" aus Geist und Natur, so dass der Ort seines Ichs, der Ort des Subjekts, von dem es aus spricht, unauslotbar bleibt: „Bald in einem Kopf, bald in einem Bauch, [...] irgendwo im Ungewissen.“ (Beckett 1995, 91)

Die Frage nach dem Zusammenhang von Liebe und Sprache, die wir hier, zumindest akzentweise, $\mathrm{zu}$ beantworten suchen, ist grundsätzlich ,eine Frage des Außen und Innen“ (Freud 1999i, 13), der Vermitteltheit von Subjekt und Objekt - oder besser gesagt: eine Frage des Verschlingungszusammenhangs. Beide, Sprache und Liebe, haben ihren Ausgangspunkt im Mund. Es ist der Mund, der spricht. Es ist der Mund, der isst. Es ist der Mund, der küsst. Im „Unbehagen in der Kultur“ schreibt Freud, dass er seine Trieblehre anfänglich auf „den Satz des Dichterphilosophen Schiller“ gestützt habe, auf den Schlussvers aus dem Gedicht „Die Weltweisen“, in dem es heißt, „daß „Hunger und Liebe“ das Getriebe der Welt zusammenhalten“. Während der Hunger, so Freud, „als Vertreter jener Triebe gelten [könne], die das Einzelwesen erhalten wollen“, dienen

1 „Die geistige Bildung, der moderne Verstand bringt im Menschen diesen Gegensatz hervor, der ihn zur Amphibie macht, indem er nun in zwei Welten zu leben hat, die sich widersprechen, so daß in diesem Widerspruch nun auch das Bewußtsein sich umhertreibt und, von der einen Seite herübergeworfen zu der anderen, unfähig ist, sich für sich in der einen wie in der anderen zu befriedigen“ (Hegel 1986, 80 f.). 
die „aufs Objekt gerichteten ,libidinösen“ Triebe der Liebe“ (Freud 1999h, 476 f.) nicht nur dem „Ichtrieb“, der egozentrischen Selbstbewahrung, sondern auch der Eigenerweiterung, dem erotischen Zusammenschluss. Trotzdem - und hier wird es dialektisch - hat jedoch auch die Liebe ihr Urbild im Hunger, denn in der frühkindlichen oralen Phase werden die Objekte der Begierde im buchstäblichen Sinn verinnerlicht: Es kommt, schreibt Freud, zum „Sicheinverleiben oder Fressen [derselben], eine Art der Liebe, welche mit der Aufhebung der Sonderexistenz des Objekts vereinbar ist, also als ambivalent bezeichnet werden kann" (Freud 1999e, 231). Der Sprachgebrauch hat dieses erste Urteil der Liebe aufgehoben, um den kannibalischen Akt im Sprachspiel zu negieren: konserviert, sublimiert, transzendiert. Geblieben sind vor allem die Redensarten: Wer liebt, verzehrt sich nach dem anderen; die Liebenden haben einander zum Fressen gern; man liebt sich mit Haut und Haar - kurz: Liebe geht durch den Magen, nicht nur wegen der Schmetterlinge im Bauch. Freud stellt sogar die These auf, dass alle Urteilsfunktionen, die entweder bejahen oder verneinen oder einem Objekt eine Eigenschaft zuschreiben, dieser kulinarischen Fragestellung folgen, denn die Frage nach „gut oder schlecht, nützlich oder schädlich“ bedeutet in „der Sprache der ältesten, oralen Triebregungen ausgedrückt: das will ich essen oder will es ausspucken, und in weitergehender Übertragung: das will ich in mich einführen und das aus mir ausschließen. Also: es soll in mir oder außer mir sein. Das ursprüngliche LustIch will [...] alles Gute sich introjizieren, alles Schlechte von sich werfen. [...] Es ist, wie man sieht, wieder eine Frage des Außen und Innen.“ (Freud 1999i, 13)

Becketts Bild von der Liebeskarotte, die es immer wieder zu vernaschen gilt, evoziert das Bild vom Esel, dem das nutritive Objekt der Begierde am Angelhaken vor Auge und Maul gehalten wird, um weiter zu traben, um weiter zu arbeiten. Die Liebeskarotte zieht. Sie lässt einen weitermachen - bis zum Ende - und deshalb schließt der Roman auch mit diesem Wort: weitermachen. „[E]s sind Worte, es gibt nichts anderes, man muß weitermachen, [...] man muß Worte sagen, solange es welche gibt, [...] man muß weitermachen, ich werde weitermachen." (Beckett 1995, 176) Je vais continuer - dieses Kontinuum ist der Zug der Sprache, der „Hunger und Liebe“, den Grundtriebfedern des Weltgetriebes, folgt; es ist der Diskurs eines unendlichen Begehrens, welches sich nach dem anderen verzehrt, ohne ihn zur Gänze verinnerlichen, verdauen zu können, weil er sich als Sprachwesen entzieht. „Als elende, vergebliche Wörter des elenden, alten Geistes habe ich die Liebe erfunden, [...] um mir zu entgehen“ (Beckett 1995, 22), bekennt der Namenlose. Die Liebe soll dazu dienen, dem Ich zu entkommen, gleichzeitig nährt sie aber auch das Individuum, das narzisstische Ego, hält es am Laufen, am Sprechen, am Sich-Selbst-Versprechen. Es ist das Versprechen der Sprache, nicht allein zu sein, das Versprechen, ,von überallher nach überallhin“ Verbindungsbrücken schlagen zu können, durch die „Zauberkraft“ des logos, der die 
primär-narzisstische Weltverschmolzenheitserfahrung, das „ozeanische Gefühl“, in der Idee der Menschheit aufhebt. Der Diskurs des Namenlosen, der nicht aufhören kann, nicht aufzuhören (wie Lacan den Todestrieb charakterisiert hat), folgt dem Zug des Begehrens: einer Sprache, die über sich selbst hinausspricht. Die Stimme des anderen, die die Dunkelheit der Selbstversunkenheit erhellt, ist die größte Liebeskarotte, der größte Köder am Angelhaken des Begehrens. Vernascht kann immer nur der Körper des anderen werden, sein Augapfel, sein Erdbeermund; was widersteht, ist der Geist der Sprache, das Skelett der Grammatik, die unheimliche Stimme der Ver-Anderung, die - als Extimes - insistiert. Eine Karotte ist keine Karotte ist keine Karotte ... anders gesagt: Eine Karotte ist eine Rose ist eine Zigarre ist das Ei, das der untote Hund, der in die Küche kommt, dem Koch immer wieder entwendet, um von ihm immer wieder tot-, entzweigeschlagen zu werden - „nach Belieben, nach Belieben“ (Beckett 1995, 127). Die Sprache zieht, zieht hinein in die unendliche Metonymie des Begehrens, in das „Liebesrezidiv“, das im Zentrum der psychoanalytischen Aufmerksamkeit steht, ${ }^{2}$ in die Suche nach der verlorenen Lust, in ihre Wiederholungen, ihre Surrogatsreihen und Supplementenketten. „An der Frauenbrust treffen sich Hunger und Liebe“ (Freud 1999b, 211), kommentiert Freud in der „Traumdeutung“ einen seiner Träume, in dem er in die Küche geht, hungrig nach Mehlspeisen, und auf eine Wirtin trifft, die seiner Mutter gleicht und die ihn ermahnt, seinen Appetit zu zügeln, da das Essen noch nicht fertig sei. Triebverzicht leisten - die Lust aufschieben - die Gier unterdrücken: Das ist der Ursprung aller Kultur, aber auch des Unbehagens in ihr, denn die Psychoanalyse lehrt, dass es dem Menschen unmöglich sei, ganz auf eine „einmal gekannte Lust“ zu verzichten; der Mensch suche danach, sie ein Leben lang wiederzufinden, zu wiederholen, in welcher entstellten oder sublimierten Form auch immer. Letztendlich ist Kultur aufgehobener Kannibalismus. Oder mit einem bösen Aphorismus Lichtenbergs gesagt: „Wir fressen einander nicht, wir schlachten uns bloß.“ (Lichtenberg 1992, K 224) In Freuds Urhorden-Mythos aus „Totem und Tabu“ verspeist die Bruderschaft den ermordeten Urvater, um durch

2 „In einem Liebesrezidiv vollzieht sich der Prozeß der Genesung, wenn wir alle die mannigfaltigen Komponenten des Sexualtriebes als ,Liebe‘ zusammenfassen, und dieses Rezidiv ist unerläßlich, denn die Symptome, wegen deren die Behandlung unternommen wurde, sind nichts anderes als Niederschläge früherer Verdrängungs- und Wiederkehrkämpfe und können nur von einer neuen Hochflut der nämlichen Leidenschaften gelöst und weggeschwemmt werden. Jede psychoanalytische Behandlung ist ein Versuch, verdrängte Liebe zu befreien, die in einem Symptom einen kümmerlichen Kompromißausweg gefunden hatte. Ja, die Übereinstimmung mit dem vom Dichter geschilderten Heilungsvorgang in der Gradiva erreicht ihre Höhe, wenn wir hinzufügen, daß auch in der analytischen Psychotherapie die wiedergeweckte Leidenschaft, sei sie Liebe oder Haß, jedesmal die Person des Arztes zu ihrem Objekte wählt.“ (Freud 1999j, 118) 
diesen Bund das erste totemistische Gesetz zu etablieren, dass keiner mehr seinen Nächsten überfallen und auffressen soll. Und Freud erwähnt auch kurz, dass in der christlichen Eucharistie und der Agape, dem Liebesmahl, diese Urgeschichte des Kannibalismus auf höchster, sublimster Ebene aufgehoben wurde (vgl. Freud 1999k, 186). Das Wort ist Fleisch geworden: Tatsächlich sind - Freuds hysterischer Konversionstheorie zufolge - alle unsere Körper durchdrungen vom Wort, von den Redensarten und Sprechweisen, von all der physiologischen Metaphorik, die uns ins Gesicht schlägt oder unser Herz tanzen, springen, brechen lässt. Diese Transsubstantiation durch den logos hat den nackten Hunger, die sowohl sexuell als auch kulinarisch konnotierte „Fleischeslust“ (Beckett 1995, 62), in das Begehren verwandelt - und damit in ein Begehren der Sprache.

Wovon man nicht essen darf, darüber muss man sprechen: Becketts Diskurs ist deshalb auch der Diskurs eines Hungerkünstlers; das Motiv der Nahrung durchzieht den ganzen Roman. ${ }^{3}$ Der Namenlose - beziehungsweise Mahood, einer seiner „Stellvertreter“- wohnt neben einem Schlachthof, in unmittelbarer Nähe einer „Pferdefleischverwertung“, und ist mit der Wirtin einer Garküche liiert, in deren Gemüsegarten er mit Blick auf die Speisekarte haust: „Hier tötet und ißt man“ (Beckett 1995, 74), lautet seine lapidare Zusammenfassung. Er nennt sich selbst einen „Hungerleider“, er sei ein „Krüppelrumpf im Napf“ (Beckett 1995, 39), der nicht nur mit „Kalbslunge“ und „Markknochen“ (Beckett 1995, 57) gefüttert werde, sondern auch mit „Redensarten“. Er sagt, sie hätten ihm „eine Sprache eingetrichtert“ (Beckett 1995, 52), eine allzu familiäre, inzestuöse Sprache, die „Liebe zum Nächsten“ fordert und vom „Schoß der Vernunft“ spricht, eine allzu verlogene, hohe Sprache, changierend zwischen „Kauderwelsch“ und „Oberprimanergerede“, die ihm nicht schmeckt, weshalb er ihre „Redensarten“ wieder herauskotzen möchte (Beckett 1995, 68). Er verlangt stattdessen nach einer einfacheren ,intellektuellen Nahrung“, die sich mit basalen Geschmacksurteilen - ganz im Sinn von Freuds oralem Beurteilungsvermögen - begnügt: „Was eine Rübe ist, weiß ich ungefähr, auch was eine Karotte ist, vor allem die zarte, auch Möhrchen genannte.“ (Beckett 1995, 58) Doch die Sprache geht über diese differenzierende Prädikation der Dinge hinaus. Es sind die fremden Stimmen, die den Namenlosen quälen, das fremde Gemurmel, das ihn „würgt“, weil man es,

3 Auch in Becketts „Endspiel“ ist der Hunger/Liebes-Topos zentral und mit dem Verfall der Sprache verquickt; es wird nur noch „geschwatzt“ - und auch die Liebe „schmatzt“ nur noch: „Was in diesem Ambiente der Humanität fortwest: daß die beiden Alten [gemeint sind Hamms Eltern Nell und Nagg, die in Mülltonnen hausen] den letzten Zwieback miteinander teilen, wird durch den Kontrast zur transzendentalen Bestialität abstoßend, der Rückstand der Liebe zur schmatzenden Intimität. Soweit sie noch Menschen sind, menschelt es.“ (Adorno 1974, 302; siehe hierzu auch: Just 2018, 234 ff.) 
wie er behauptet, in ihn „hineingestopft“ (Beckett 1995, 68) habe. Er ist mit der Sprache der anderen gemästet, überfüttert, bis oben hin voll. Wenn die Liebe, wie es bei Beckett heißt, eine Karotte ist, die immer zieht, so ist damit auch die Sprache gemeint, die verlockt, verführt, einen in Bann schlägt, gefesselt hält. Une carotte - von diesem Wort ist es, zumindest im Lautlichen, nicht weit zum französischen garrotter - knebeln, mundtot machen - und damit zur Garrotte, dem Mord- und Hinrichtungswerkzeug, dem Würgeeisen, das erdrosselt, stranguliert. Eine Karotte: eine Garrotte. Die Liebeskarotte ist immer auch die Sprach-, die Wortgarrotte. Die Liebe zieht, zieht an; die Sprache würgt, fesselt, schneidet wie die Liebe ins Fleisch. Ein brutales Möbiusband: Die Artikulation des Begehrens ist das Begehren der Artikulation. Deshalb ist der Protagonist auch mit einem Ring um den Hals an den Krug, in dem er haust, angebunden. Es ist sein „Halsband“, er spricht von seinem „Pranger“ aus (Beckett 1995, 63). Wenn wir gesagt haben, dass das mächtigste Medium bzw. Instrument des Eros die Sprache sei, weil sie alles mit allem, auch mit dem Fernsten und Fremdesten verbinden kann, dann muss man dieses mächtige Band auch kritisch sehen. Das Subjekt nach Freud, Lacan und Beckett ist der Sprache unterworfen: Sub-jectus. Die Sprache ist ihm von Geburt an „eingetrichtert“, in ihn „hineingestopft“ worden, so dass er an ihr „würgt“, sie wieder „erbrechen“ muss. Der unaufhörliche Diskurs, den Becketts Namenloser von sich gibt, steht deshalb auch unter dem Zeichen der Folter: „Ich bin [...] da, um gepeinigt zu werden“ (Beckett 1995, 50), heißt es an einer Stelle; und etwas später: „Man kann sich nur noch in aller Ruhe auf die Folter strecken, in der Wonne, zu wissen, auf ewig niemand zu sein.“ (Beckett 1995, 72) Im Gemurmel, im Gerede, im polyphonen Stimmengewirr der anderen entgrenzt sich das Subjekt, wird namenlos, entzieht sich, indem es versucht, den Identitäten, denen es durch die Eintrichterungen des Muttersprachenbreis, der immer auch ein ideologischer Schwedentrunk ist, ${ }^{4} \mathrm{zu}$ entkommen. Die Sprache foltert, richtet zu, sie schneidet ins Fleisch, würgt ab. Genauso wie es die Liebe tut: Denn durch die neuen Verbindungen, die das Eros-Prinzip anstrebt, geht auch immer etwas an dem, was sich da zu größeren Einheiten verbindet, zugrunde. Wenn Spinozas Satz gilt, omnis determinatio est negatio, dann gilt auch omnis copulatio est negatio. Slavoj Žižek hat darauf hingewiesen, dass Lacan gegen Heidegger eingewandt habe, dass das „Haus der Sprache“, welches der Mensch bewohnt, kein gemütliches sei, in dem man es sich leicht einrichten könne - im Gegenteil: es sei ein

4 Der sogenannte Schwedentrunk war eine während des Dreißigjährigen Krieges häufig angewandte Foltermethode, bei der dem Opfer durch einen Trichter Jauche oder eine andere abstoßende Flüssigkeit in den Mund eingeführt wurde. 
„Folterhaus der Sprache“ (Žižek 2012). ${ }^{5}$ „In der Freudschen Perspektive“, zitiert Žižek Lacan, „ist der Mensch das von der Sprache erfaßte und gepeinigte Subjekt.“ (Žižek 2014, 1183) Das Bild von der Karotte, das am Gängelband der Sprache den verliebten Esel antreibt, evoziert diese Pein: Die unüberbrückbare Kluft zwischen dem, was das Subjekt begehrt, und dem, was es zu erreichen vermag, ist auch der unaufhebbare Einschnitt zwischen Subjekt und Sprache. Die Schlinge der Sprache, ihr Verschlingungszusammenhang, nötigt dazu, sich aus ihrem unmittelbaren Zugriff zu befreien, was eben nur durch die Subtraktion von der gewöhnlichen Sprache funktioniert, sei es die des Alltags mit ihren dümmlich-brutalen Sprichwort-Weisheiten - „wer zuerst kommt, mahlt zuerst“; „alles hat ein Ende, nur die Wurst hat zwei“ - oder auch des leeren Sprechens, jenes übliche Bescheidwissen im bildungsbürgerlichen oder akademischen Pfauenradschlag. Der Namenlose fühlt sich von der Sprache gefoltert, er nimmt die Folter auf sich, um nun seinerseits den Diskurs zu deformieren. Žižek verweist in diesem Zusammenhang auf Elfriede Jelineks Forderung „Man muß die Sprache foltern, damit sie die Wahrheit sagt.“ (Jelinek 1995, 73) Die Suche nach dem wahren Wort, der sich die Literatur verschrieben hat, ist sich der einschneidenden Kraft der Sprache bewusst. Sie muss die Gewalt der Sprache auf sich nehmen, um sie in eine revolutionäre zu verwandeln. Als zusätzliche Referenz sei hier nur Kafka genannt, der an Milena schrieb, dass er sich „mit nichts anderem als mit Gefoltert-werden und Foltern“ beschäftige, „um aus dem verdammten Mund das verdammte Wort zu erfahren“ (Kafka 1986, 290), oder Peter Handkes Kaspar Hauser-Stück: „Es zeigt“, merkte dazu Handke an, ,wie jemand durch Sprechen zum Sprechen gebracht werden kann. Das Stück könnte auch ,Sprechfolterung‘ heißen.“ (Handke 1973, 7) Mehr noch: Die ganze avancierte Literatur - Kafka, Joyce, Beckett, Proust, Gombrowicz - könnte als eine solch semiologische tour de torture charakterisiert werden. Sich von der grammatologischen Garrotte, dem ererbten Würgeband der Sprache befreien, indem man sie so entstellt, so verwendet, dass - wie es Deleuze mit Bezug auf Marcel Proust genannt hat - ,eine Art Fremdsprache“, ein „AndersWerden der Sprache“ (Deleuze 2000, 16) möglich wird, ein erlösendes Delirium, befreit vom überkommenen ideologischen Zwang: „Wenn ich ein Wort höre“, erläuterte Jelinek in einem Interview, „muss ich sofort Alliterationen, Paraphrasen, Metathesen herstellen, Silben vertauschen. [...] Es ist [...] ein sehr alter Wunsch, die Sprache selbst zu zwingen, die Wahrheit zu sagen und ihren Ideologiecharakter preiszugeben. “ (Jelinek 1995, 72f.) Die Ent-Stellung der Sprache ist notwendig: „Man muß versuchen, die Dinge so zu umschreiben, sie so zu ver-

5 Vgl. hierzu auch den Abschnitt „Im Folterhaus der Sprache“ in „Weniger als Nichts“ (Žižek 2014, 1182ff.) 
zerren, daß sie zur Kenntlichkeit entstellt werden.“(Jelinek 1995, 49) Was Jelinek hier anspricht, ist die polymorph-perverse Poetik der Sprache, die grenzenlose Kopulationskraft ihrer Worte, denn jedes Wort lässt sich über das metonymischmetaphorische Band, über die sinnlich-semantischen Assoziationen, mit allen anderen Wörtern verbinden, so wie die Karotte auch die Garrotte ist und so wie im Esel, den die Karotte zum Weitermachen verlockt, auch - von hinten genommen und als Palindrom erkannt - der literarische Imperativ schlechthin steckt: Lese! Die polymorph-perverse Entstellung der Worte kennt keine Grenzen und damit auch die Entstellungen des Subjekts, das - im „Folterhaus der Sprache“ gefangen - die Worte gebraucht und missbraucht, indem es von ihnen gebraucht und missbraucht wird, auf der Suche nach der Wahrheit, nach dem ,verdammten Wort aus dem verdammten Mund“, nach dem Namen des Namenlosen. Die Arbeit am Begriff, die sowohl die Philosophie als auch die Literatur verrichtet, erfolgt im Namen des Eros: Die Wahrheit der Sprache liegt in der Idee der Menschheit, im universalen Band einer Gerechtigkeit, die auch den Letzten, das geringste, namenlose Abjekt, mit einschließt. Denn Literatur und Philosophie richten sich immer an alle, egal wie „gefoltert“, entstellt, zerstückelt, verwickelt oder deformiert ihre Sprache auch sein mag - im Gegenteil: Es ist gerade diese gewaltsame Zurichtung, die an die potenzielle Freiheit jeder Leserin und jedes Lesers appelliert, es auch verstehen zu können. Es - das ist die Wahrheit. In dieser Hinsicht hat Roland Barthes einmal von einer „Erotik des lesbaren Satzes“ gesprochen, von einer „Nächstenliebe des Thetischen“, von einer „Agape der Syntax“: „Man kann nie genug betonen, wieviel Liebe (zum andern, zum Leser) in der Arbeit am Satz steckt.“ (Barthes 2006, 383) Das mag - Roland Barthes hat das auch selbstkritisch angemerkt - wie eine „humanistisch abgeschmackte“ Idee klingen, aber sie verliert ihre Abgeschmacktheit, wenn man darauf beharrt, darauf insistiert, dass in dieser „Agape der Syntax“, diesem literarischen Liebesmahl, die Gewalt der ganzen Sprache mitschwingt, jene unaufhebbare Unheimlichkeit, von der das „Folterhaus der Sprache“ durchdrungen ist. Es geht um die Notwendigkeit, dieser entstellenden Gewalt der Sprache, ihrer Negativität ins Auge zu sehen. Es geht um die Erkenntnis, dass - wenn die Sprache die Liebesgabe der Wahrheit sein soll im Lesbaren auch immer ein Unlesbares, ein Unverdauliches, stecken muss.

Zuletzt, sozusagen als Schlussstein unserer Reflexionen, sei noch an ein antikes mythologisches Bild erinnert, das auch bei Beckett wiederkehrt. Gemeint ist Molloy, der Protagonist des ersten Teils der Beckett'schen Romantrilogie, der auch im „Namenlosen“ erwähnt wird und der es liebt, Kieselsteine zu lutschen: „Es waren kleine Kiesel, aber ich nenne sie Steine. [...] Ich verteilte sie gleichmäßig in meinen vier Taschen und lutschte sie nacheinander.“ (Beckett 1995b, 95) In der Mundhöhle treffen sich Hunger, Liebe und Sprache. Der Mund: er beißt, er küsst, er saugt, er spricht. Die Dialektik der sprachlichen Gewalt ist eine von 
Zunge und Zahn, wie sie der griechische Mythos von Kronos darstellt: Kronos, der grausame Titan, der perverse Urvater, frisst seine Kinder und erbricht sie erst wieder - lebendig -, nachdem man ihm statt Fleisch einen Stein zum Essen gegeben hat. Der Stein der Auferstehung steht freilich für den logos, für das unaufzehrbare, unverdauliche Wort, das den Verschlingungszusammenhang der nackten Lebensnot, den ewigen natürlichen Kreislauf des Fressen-und-GefressenWerdens unterbricht, ihn auf den Geist, die Erkenntnis des höchsten Zusammenhanges hin öffnet. In diesem Sinn spricht alle Literatur von Hunger und von Liebe; in dieser Hinsicht ist der Diskurs der Literatur eine große, gewaltige und niemals abzuschließende Kronos-Dekonstruktion.

\section{Literaturverzeichnis}

Adorno, Theodor W. „Versuch, das Endspiel zu verstehen“. Noten zur Literatur II, Gesammelte Schriften Bd. 11. Frankfurt/M.: Suhrkamp, 1974.

Barthes, Roland. Das Rauschen der Sprache. Frankfurt/M.: Suhrkamp, 2006.

Beckett, Samuel. Der Namenlose. Frankfurt/M.: Suhrkamp, 1995.

Beckett, Samuel. Molloy. Frankfurt/M.: Suhrkamp, 1995b.

Deleuze, Gilles. Kritik und Klinik. Frankfurt/M.: Suhrkamp, 2000.

Freud, Sigmund. „Über Deckerinnerungen“. Gesammelte Werke Bd. I. Frankfurt/M.: Fischer, 1999a.

Freud, Sigmund. „Die Traumdeutung“. Gesammelte Werke Bd. II/III. Frankfurt/M.: Fischer, 1999b.

Freud, Sigmund. „Drei Abhandlungen zur Sexualtheorie“. Gesammelte Werke Bd. V.

Frankfurt/M.: Fischer, 1999c.

Freud, Sigmund. „Zeitgemäßes über Krieg und Tod“. Gesammelte Werke Bd. X. Frankfurt/M.: Fischer, 1999d.

Freud, Sigmund. „Triebe und Triebschicksale“. Gesammelte Werke Bd. X. Frankfurt/M.: Fischer, 1999e.

Freud, Sigmund. „Vorlesung zur Einführung in die Psychoanalyse“. Gesammelte Werke Bd. XI. Frankfurt/M.: Fischer, 1999f.

Freud, Sigmund. „Massenpsychologie und Ich-Analyse“. Gesammelte Werke Bd. XIII. Frankfurt/M.: Fischer, 1999g.

Freud, Sigmund. „Das Unbehagen in der Kultur“. Gesammelte Werke Bd. XIV. Frankfurt/M.: Fischer, 1999h.

Freud, Sigmund. „Die Verneinung“. Gesammelte Werke Bd. XIV. Frankfurt/M.: Fischer, 1999i.

Freud, Sigmund. „Der Wahn und die Träume in W. Jensens ,Gradiva““. Gesammelte Werke Bd. VII. Frankfurt/M.: Fischer, 1999j.

Freud, Sigmund. „Totem und Tabu“. Gesammelte Werke Bd. IX. Frankfurt/M.: Fischer, 1999k. Handke, Peter. Kaspar. Frankfurt/M.: Suhrkamp, 1973.

Hegel, Georg Wilhelm Friedrich. Vorlesungen über die Ästhetik I. Frankfurt/M.: Suhrkamp, 1986.

Jelinek, Elfriede, Jutta Heinrich und Adolf-Ernst Meyer. Sturm und Zwang - Schreiben als Geschlechterkampf. Hamburg: Klein, 1995. 
Just, Rainer. Der Tod, die Liebe, das Wort - Zum literarischen Komplex der Psychoanalyse. Wien: Klever, 2018.

Kafka, Franz. Briefe an Milena. Frankfurt/M.: Fischer, 1986.

Lacan, Jacques. Encore - Das Seminar Buch XX. Weinheim und Berlin: Quadriga, 1986.

Lichtenberg, Georg Christoph. „Sudelbücher“. Schriften und Briefe Bd. I. Frankfurt/M.: Insel, 1992.

Žižek, Slavoj. „Dichter und Henker“. Tages-Anzeiger, 2.11.2012.

Žižek, Slavoj. Weniger als nichts - Hegel und der Schatten des dialektischen Materialismus. Berlin: Suhrkamp, 2014.

Rainer Just, geboren 1971 in Wien, Studium der Vergleichenden Literaturwissenschaft und der Deutschen Philologie, lehrt seit 2004 Literaturtheorie am Institut für Vergleichende Literaturwissenschaft der Universität Wien. Daneben diverse essayistische und literarische Tätigkeiten. Zuletzt: Der Tod, die Liebe, das Wort Zum literarischen Komplex der Psychoanalyse, Wien 2018. 